\title{
Growth mechanism of $\mathrm{ZnO}$ nanostructures for ultra-high piezoelectric $d_{33}$ coefficient
}

Moumita Ghosh ${ }^{a}$, Mohan G. Rao ${ }^{b^{*}}$

${ }^{a}$ Centre for Nano Science and Engineering, Indian Institute of Science, Bangalore, India.

${ }^{\mathrm{b}}$ Department of Instrumentation and Applied Physics, Indian Institute of Science, Bangalore, 560012 India.

AUTHOR INFORMATION

Corresponding Author

* E-mail: gmrao@isu.iisc.ernet.in; Tel: +91 802293 2349; Fax: +91 8023600135. 


\section{ABSTRACT:}

A comparative morphological study of different $\mathrm{ZnO}$ nanostructures was carried out with different varying process parameters for energy harvesting. Molarity, temperature, growth duration and seed layer were such fundamental controlling parameters. The study brings out an outstanding piezoelectric coefficient $\left(\mathrm{d}_{33}\right)$ of $44.33 \mathrm{pm} / \mathrm{V}$ for vertically aligned $\mathrm{ZnO}$ nanorod structures, considered as the highest reported $\mathrm{d}_{33}$ value for any kind of $\mathrm{ZnO}$ nanostructures. XRD analysis confirms wurtzite nature of this nanorod structure with [0001] as preferential growth direction. Semiconducting characteristic of nanorods was determined with temperature induced I/V characterization.

KEYWORDS: ZnO, nanostructures, scanning electron microscopy (SEM), X-ray diffraction (XRD), Piezoelectric Force Microscopy (PFM), Current/voltage (I/V) characteristic.

\section{INTRODUCTION}

In recent years, $\mathrm{ZnO}$ based nanostructures have attracted attention due to their application in diverse fields. Wide direct band gap of $\mathrm{ZnO}$ along with proper tuning by doping has created interesting application in optics [1, 2] and electronics [3-6]. Doping with different materials is also successful in creating properties like ferromagnetism [7, 8] and ferroelectricity $[9,10]$ in $\mathrm{ZnO}$ at nanoscale. Electromechanical property of environment friendly $\mathrm{ZnO}$ has a sound impact in the field of sensors and actuators, like biological sensing [11, 12], gas sensing [13, 14] and especially in energy harvesting [15-19]. Our objective is to grow vertically aligned [0001] directed $\mathrm{ZnO}$ nanorods by low temperature sol-gel method for energy harvesting application. In this process, we explored the evolution of nanostructured morphologies in relation to the process parameters. Here, we report on the synthesis of different $\mathrm{ZnO}$ morphologies at nanoscale with a simple low-cost hydrothermal/sol-gel method at atmospheric pressure. Sol-gel method is 
preferred because of its certain advantages over other conventional techniques (such as CVD [20, 21], VLS [22, 23], PLD, magnetron sputtering, and MOVPE [24]). It does not require high vacuum, sophisticated equipment, and high temperature. Beside all, the growth process is easily controllable with high reproducibility. We studied the influence of various process parameters like, molarity of chemical reagents, process temperature, process time and the seed layer on the microstructure evolution. These parameters facilitate to obtain different growth morphology of $\mathrm{ZnO}$ nanostructures. Finally, we obtained vertically aligned $\mathrm{ZnO}$ nanorods and $\mathrm{XRD}$ analysis confirms [0001] orientation of this nanostructure. Current-voltage (I/V) measurement helps in understanding the behavior of vertically aligned $\mathrm{ZnO}$ nanorods. We have used Piezoelectric Force Microscopy (PFM) as a tool for electro-mechanical characterization of the nanorod structures. This characterization showed maximum value of piezoelectric coefficient of $\mathrm{ZnO}$ nanostructures till date.

\section{EXPERIMENTAL}

\subsection{Material synthesis}

Zinc-nitrate-hexahydrate $\left[\mathrm{Zn}\left(\mathrm{NO}_{3}\right)_{2} \cdot 6 \mathrm{H}_{2} \mathrm{O}\right]$ and hexamethylene-tetramine $\left[\mathrm{C}_{6} \mathrm{H}_{12} \mathrm{~N}_{4}\right]$ were the reagents used to obtain $\mathrm{ZnO}$ nanostructures. They were mixed in 1:1 molar ratio. This solution was kept under constant temperature and ultrasonicated for two hours. The ultrasonication allows the solution to become homogeneous. This was transferred into a semi-closed Pyrex bottle. Substrates like Si and $30 \mathrm{~nm}$ thick ZnO seed layer coated Pt/Si (100) were already prepositioned in the Pyrex bottle. The solution was then treated under atmospheric pressure for 1.5 hour to 5 hour. The temperature of process solution was varied from $95^{\circ} \mathrm{C}$ to $150{ }^{\circ} \mathrm{C}$. After the hydrothermal process, the substrates were washed with DI water and ethanol repeatedly and 
dried with nitrogen blower. All the samples were dried at $150^{\circ} \mathrm{C}$ for two minutes. We have used different concentrations of precursor in our experiment starting from $1 \mathrm{mM}$ to $600 \mathrm{mM}$. The reactants were mixed in 1:1 ratio.

\subsection{Characterization}

For ZnO morphology evaluation, we used high vacuum SEM (Ultra 550 FESEM, Carl Zeiss AG, Oberkochen, Germany, with pressure less than $\left.10^{-5} \mathrm{mbar}\right)$. The accelerating voltage of electron during characterization was around 3KV. The working distance (WD) was less than $8 \mathrm{~mm}$. For crystallographic analysis, D8 Advance XRD (Bruker AXS GmbH, Karlsruhe, Germany), with Copper $\mathrm{K}_{\alpha}$ radiation $(\lambda=1.540598 \AA)$ was used.

Electrical characterization of the vertically aligned nanostructured thin-film was carried out with Agilent Device Analyzer B1500A with thermal chuck (Agilent Inc., Santa Clara, California, US). Current-voltage characteristics (I/V) were studied at different temperatures starting from $27^{\circ} \mathrm{C}$ to $200^{\circ} \mathrm{C}$.

Piezoelectric Force Microscopy (PFM) mode was used (Dimension Icon AFM, Bruker AXS GmbH, Karlsruhe, Germany) to characterize the piezoelectric behavior of the aligned ZnO nanostructures. PFM was operated in vertical mode with an AC driving voltage of $15 \mathrm{~V}$. The driving frequency was $60 \mathrm{KHz}$. Here, SCM-PIT tips with tip bias $-12 \mathrm{~V}$ to $+12 \mathrm{~V}$ were used.

\section{RESULTS AND DISCUSSION}

\subsection{Effect of precursor concentration}

We observed a rapid change in nanostructure morphology with change in precursor concentration. Figure 1 shows false color SEM images of different ZnO nanostructure 
morphologies. They were grown on Si substrates at a constant temperature of $150^{\circ} \mathrm{C}$ for 5 hours. The shapes of these morphologies are like, nanowires (figure 1(a)), miss-aligned cylindrical standing (figure 1(b)), rice like structures (figure 1(c)), dense rice bed (figure 1(d)), nanoflower (figure 1(e)), porous sponge (figure 1(f)) and porous spongy carpet (figure 1(g)). These show drastic change in $\mathrm{ZnO}$ nanostructures with change in concentration of precursor solution, while other process parameters were kept constant. We used different molarities of precursor solutions for experiment. These were $1 \mathrm{mM}$ (figure 1(a)), $10 \mathrm{mM}$ (figure 1(b)), $25 \mathrm{mM}$ (figure 1(c)), 50 $\mathrm{mM}$ (figure 1(d)), $100 \mathrm{mM}$ (figure 1(e)), $500 \mathrm{mM}$ (figure 1(f)) and $600 \mathrm{mM}$ (figure 1(g)). Table 1 shows average size of different nanostructures.

Table 1. Average size of different nanostructures:

\begin{tabular}{l|cccc}
\hline & $\begin{array}{c}\text { End } \\
\text { diameter } \\
(\mathbf{n m})\end{array}$ & $\begin{array}{c}\text { Center } \\
\text { diameter } \\
(\mathbf{n m})\end{array}$ & $\begin{array}{c}\text { Length } \\
\text { ( } \boldsymbol{\mu m})\end{array}$ & $\begin{array}{c}\text { Wall } \\
\text { thickness } \\
(\mathbf{n m})\end{array}$ \\
\hline Nanowire & 200 to 250 & N.A. & $>50$ & N.A. \\
\hline Miss-aligned cylindrical standing & 100 to 150 & N.A. & 1 to 1.5 & N.A \\
\hline Rice like structures & 55 to 65 & 150 to 200 & 1 to 1.2 & N.A. \\
\hline Dense rice bed structures & 40 to 50 & 70 to 80 & 1.2 to 1.5 & N.A. \\
\hline Nanoflower & 100 to 150 & N.A. & 1 & N.A. \\
\hline Porous sponge & N.A. & N.A. & N.A. & 40 to 50 \\
\hline Porous spongy carpet & N.A. & N.A. & N.A. & 100 to 150 \\
\hline
\end{tabular}

*N.A. refers not applicable.

Thermodynamically, hexagonal wurtzite structure is the most stable structure of $\mathrm{ZnO}$ [25]. This structure (figure 2(a)) has highest surface energy in polar $c$ planes $(0001) /(000 \overline{\mathbf{1}})$. In order to minimize surface energy, growth occurs along $<0001>\mid<000 \overline{\mathbf{1}}>$ directions. This gives

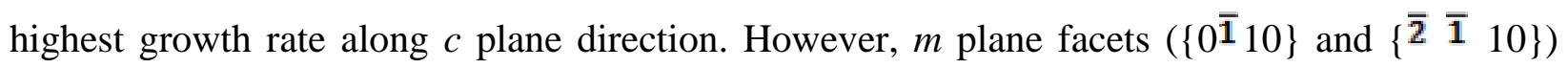
also grow. This contributes bulky centered feature. In low temperature hydrothermal growth under constant temperature and atmospheric pressure, $\mathrm{ZnO}$ nuclei are formed from 
supersaturated solution of $\mathrm{Zn}\left(\mathrm{NO}_{3}\right)_{2} \cdot 6 \mathrm{H}_{2} \mathrm{O}$ and $\mathrm{C}_{6} \mathrm{H}_{12} \mathrm{~N}_{4}$. With increase in concentration, supersaturation of the process solution increases. To reduce the supersaturation, multiple numbers of $\mathrm{ZnO}$ nuclei start forming. So, in short, increasing molarity initiates nuclei formation at a faster rate. These nuclei are joined together and form a large $\mathrm{ZnO}$ seed. Growth proceeds with successive attachment of $\mathrm{O}^{2-}$ and $\mathrm{Zn}^{2+}$ ions to the activation sites of seed. Figure 2(b) illustrates formation of different $\mathrm{ZnO}$ seed. Figure 1 (a) to (e) shows morphologies of different nuclei formation for variable concentration. When the concentration of reaction solution is very high, seeds are formed from highly supersaturated solution. This gives rise to formation of 2D sheet like structure (figure 2(b)). Increasing time accumulates these 2D sheets into porous spongy structure (figure 1 (f) and (g)). So, increase in precursor concentration transforms 1D structure into 2D structure (nano wire to nano carpet). Leung et al., [26, 27] has observed similar behavior while synthesizing different $\mathrm{ZnO}$ nanostructures by electrodeposition.

\subsection{Effect of synthesis temperature}

Process temperature plays an important role on the morphology. Change in $\mathrm{ZnO}$ nanostructure is observed with enhancement of process temperature (figure 3 (a) - (c)). As the temperature increases from $95^{\circ} \mathrm{C}$ to $120^{\circ} \mathrm{C}$, number of rice like morphological features and their length $(1000 \mathrm{~nm}$ to $1500 \mathrm{~nm})$ increases. But it does not influence the diameter $(40-50 \mathrm{~nm})$ of the nanostructures effectively. The experiment took place with other constant process parameters (such as $50 \mathrm{mM}$ process solution, duration five hours and Si substrate). Finally, at $150^{\circ} \mathrm{C}$ highest density of rice bed is observed. Some of the rice structures tend to give complex agglomerative morphology. This happens due to high temperature. 
Increasing temperature accelerates supersaturation of the solution. It supports rapid $\mathrm{ZnO}$ nuclei formation and finally, different direction oriented $\mathrm{ZnO}$ seed growth. This type of seed ultimately gives rise to joined $\mathrm{ZnO}$ nanostructures. Figure 3(a) - (c) shows the same with respect to temperature from $95^{\circ} \mathrm{C}$ to $150^{\circ} \mathrm{C}$.

\subsection{Effect of process duration}

Process time has profound effect on $\mathrm{ZnO}$ morphology variation. Figure 4 (a - c) shows the effect of growth duration (from 3 to 5 hours). Here, other parameters were kept constant (50 mM process solution at $150^{\circ} \mathrm{C}, \mathrm{Si}$ substrate). $\mathrm{ZnO}$ nanostructures start growing laterally after 3 hour. Finally, figure 4 (c) demonstrates dense rice bed structures with tapered ends. The number of structures and their length increases (from $200 \mathrm{~nm}$ to $1500 \mathrm{~nm}$ ) with time. Initially there is an increase in diameter of the structures (up to $40-50 \mathrm{~nm}$ ). But further increment in diameter is not observed from $120^{\circ} \mathrm{C}$ to $150^{\circ} \mathrm{C}$.

As the duration increases, size of $\mathrm{ZnO}$ seed also increases. If the solution has high concentration, supersaturation allows higher number of $\mathrm{ZnO}$ nuclei to be formed. This ultimately produces seed. So, with increasing process time not only nanostructures increase in size, but also their number increases. This can be observed from figure 4 (a-c). We have noticed that increasing time reduces $\mathrm{ZnO}$ formation unit and favors only $c$ plane oriented growth. This introduces tapered feature in grown structures at the end. Temperature also helps small thin nanostructures to join together. This joining takes place at $m$ place surface. Rice like shape with bulky center (figure 4 (d)) is ultimately synthesized. The figure 4(e) shows the tapered feature at both the end. This type of morphology can also be observed in figure 1 (c) and (d). 


\subsection{Effect of ZnO seed layer}

Figure 5 (a) shows highly aligned dense nanorod structures. These are grown on $30 \mathrm{~nm}$ sputtered $\mathrm{ZnO}$ seed layer. Figure 5 (b) shows the cross sectional view of the same. We notice from the image that these nanorod structures are uniformly grown and densely packed on the substrate. Density of these structures is approximately $2.6 \times 10^{10} \mathrm{~cm}^{-2}$. It is evident from the cross sectional view that these structures are grown vertically with respect to the substrate. Here, $25 \mathrm{mM}$ concentration of solution was used at $95{ }^{\circ} \mathrm{C}$ for 1.5 hour. $\mathrm{ZnO}$ seed layer enables aligned vertical direction growth. This is hard to obtain in the absence of seed layer keeping all the other constraints same. From the SEM image, we observe nanorod structures are around $500 \mathrm{~nm}$ in length and $50 \mathrm{~nm}$ in diameter. As the process temperature was increased from $95{ }^{\circ} \mathrm{C}$ to $150{ }^{\circ} \mathrm{C}$, vertical growth has terminated and lateral rice like growth gets initiated (figure 5 (c)).

In the presence of $30 \mathrm{~nm} \mathrm{ZnO}$ seed layer, $\mathrm{ZnO}$ nanostructure prefers to grow in certain orientation. This refers to preferential orientation. So, seed layer assists in directional growth. This gives rise to vertically aligned [0001] oriented ZnO nanostructure on substrate. Nucleation energy needed for a material to grow on same material is less compare to different material. So, at comparatively low temperature $\left(95^{\circ} \mathrm{C}\right)$ and in less time highly aligned, oriented dense vertical $\mathrm{ZnO}$ nanorod structure growth is possible on $\mathrm{ZnO}$ seed layer (figure 5 (a) and (b)). Rate of the nuclei formation increased, if we increase the temperature to $150^{\circ} \mathrm{C}$. This terminates growth of well aligned $\mathrm{ZnO}$ nanorods. With increasing time, larger dimension, tapered $\mathrm{ZnO}$ rod structures were formed (figure 5 (c)). They look similar to morphology on Si substrate without seed layer in figure 1 (c) but have larger dimensions. 
X-Ray Diffraction (XRD) analysis was carried out for the sputtered seed layer and aligned nanorods to confirm the orientation. Figure 6 (a) and (b) show the XRD patterns of 30 nm sputtered $\mathrm{ZnO}$ seed layer on Pt substrate and vertically aligned $\mathrm{ZnO}$ nanorods, respectively. We observe a very low intensity (002) peak in the sputtered seed layer. This influences further growth of $\mathrm{ZnO}$ in [0001] direction. XRD peak at $34.47^{\circ}$ Bragg's angle $\left(2 \theta^{\circ}\right)$ in figure (b) indicates well crystalline highly (002) directional aligned $\mathrm{ZnO}$ nanorod structures. This proves that growth from hydrothermal process follows $\mathrm{ZnO}$ seed layer's orientation. We have interpreted from JCPDS-36-1451 that growth morphology have hexagonal wurtzite phase. Presence of only $\mathrm{ZnO}$ peak other than substrate peak (Pt) confirms purity of $\mathrm{ZnO}$ compound. We have calculated lattice parameters $a$ and $c$ using standard equations [28] and grain size using Scherrer's formula [29] which is given as follows:

$d \equiv \frac{K \lambda}{\beta \operatorname{Cos}(\theta)}$

Eqn. (1)

where, $d$ is grain size of $\mathrm{ZnO}$ nanostructures in nanometer, $K(=0.9)$ is shape factor, $\lambda$ is $\mathrm{X}$-ray wavelength in $\AA$, $\beta$ is full width at half maximum (FWHM) in radian and ${ }^{\theta}$ is half of Bragg's angle in degree. Lattice parameters of the aligned nanorod structure obtained from the XRD data are $3.234 \AA$ and $5.211 \AA$. Where, grain size was $43.76 \mathrm{~nm}$. The lattice parameters are well matched with the JCPDS-36-1451. The $c / a$ ratio is 1.611 which indicates wurtzite nature of the structure. XRD data also gives information of Zn-O bond length. From standard equations [30] given by,

$L=\sqrt{\frac{a^{2}}{3}+(0.5-u)^{2} c^{2}}$

Eqn. (2) 
where, $L$ is bond length and $u=\frac{a^{\bar{a}}}{3 c^{2}}+0.25$ for hexagonal structure. Zn-O bond length is calculated and it is $1.97 \AA$. This is comparable with the theoretical Zn-O bond length [31]. It is well known that only $c$ axis oriented structures show good piezoelectric response. The other types of $\mathrm{ZnO}$ nanostructures grown in this study did not show preferential $c$ axis orientation as shown by the nanorods. So, for the purpose of electro-mechanical application vertically aligned [0001] oriented nanorods have been considered for further electrical studies.

Two probe I/V characterization was used for the vertically aligned $\mathrm{ZnO}$ nanorods. Figure 7 (a) illustrates schematic of the experiment and (b) represents $\mathrm{I} / \mathrm{V}$ characteristics of the nanorods. For electrical characterization, $\mathrm{Al}$ microelectrodes were formed on top of $\mathrm{ZnO}$ nanorod structures by thermal evaporation. These microelectrodes have diameter around $250 \mu \mathrm{m}$ and thickness nearly $150 \mathrm{~nm}$. These act as top electrode and Pt of Pt/ p-Si (100) substrate is treated as bottom electrode. From figure (b) we notice almost linear I/V characteristic which indicates that ohmic contact has established in between aligned vertical nanorods and Al top electrode. In a metal-semiconductor contact, if the work function of metal $\left(\Phi_{\mathrm{m}}\right)$ is less than the work function of the semiconductor $\left(\Phi_{\mathrm{s}}\right)$, then ohmic contact forms in between them. Here, $\Phi_{\mathrm{Al}}$ is around $4.30 \mathrm{eV}$ [32] and $\Phi_{\mathrm{ZnO}}$ is nearly $4.45 \mathrm{eV}$ [33]. Higher work function of $\mathrm{ZnO}$ than $\mathrm{Al}$ explains the reason of ohmic contact formation. Further this characteristic supports semiconducting [34] nature of $\mathrm{ZnO}$ nanorod structures. Slope of I/V curve increases for temperature variation from room temperature to $200^{\circ} \mathrm{C}$. This tells us that the electrical resistance of $\mathrm{ZnO}$ nanorod structure decreases with increasing temperature. The exponential decrease of resistance with respect to temperature is shown in figure 7(c). In semiconducting material, number of free electrons available is very low. Increase in temperature energizes electrons in valance band. These energized electrons go to high energy state. Finally, they become free 
electrons. It increases conductance of the material and decreases resistance. So, I/V characteristics indicate semiconducting nature of nanorods.

This [0001] oriented vertically aligned nanorods are aimed to be used in vibration based energy harvesting application. So, we investigated piezoelectric behavior of them with PFM. PFM gives local piezoelectric characteristic of the sample. Phase and amplitude response of the ZnO nanorod are displayed in figure 8 (a) and (b) respectively. With the varying tip bias from $12 \mathrm{~V}$ to $+12 \mathrm{~V}$, we observe $180^{\circ}$ phase shift in phase response, while the amplitude response forms a butterfly loop. We also notice a shift in both the responses towards the positive bias voltage (around $2 \mathrm{~V}-2.5 \mathrm{~V}$ ). Local piezoelectric coefficient $\left(\mathrm{d}_{33}\right)$ calculated from PFM data is $44.33 \mathrm{pm} / \mathrm{V}$ (SCM PIT tip sensitivity of $112 \mathrm{~nm} / \mathrm{V}$ ). This piezoelectric coefficient value is significantly higher than the values reported by Scrymgeour et al., [35] for undoped $\mathrm{ZnO}$ nanorods. It is also notably higher compare to the maximum value by Wang et al., [36] for different nanostructures. It is almost 4.5 times higher than that of bulk piezoelectric constant value, which is around $9.93 \mathrm{pm} / \mathrm{V}$ [36]. Previously we have reported piezoelectric coefficient of nanorod structures to be $8.4 \mathrm{pm} / \mathrm{V}$ [37]. But here we observe an increase by five times. This happens due to pure [0001] direction preferential growth of $\mathrm{ZnO}$ nanorod structures, which was absent in our earlier study. Decrease in the rod diameter can also enhance piezoelectric property [38].

\section{CONCLUSIONS}

Vertically aligned [0001] oriented $\mathrm{ZnO}$ nanorod structures are synthesized from low temperature sol-gel method. During synthesis, effect of different process parameters on the growth morphology is analyzed in detail. XRD analysis approves purity and wurtzite nature of $\mathrm{ZnO}$ nanorod structures grown on $\mathrm{ZnO}$ seed layer. It also confirms [0001] preferential growth of 
nanorods. I/V characterization under variable temperature condition shows semiconductive nature of $\mathrm{ZnO}$ nanorod structure. To use this nanostructure in energy harvesting application, piezoelectric coefficient measurement was very much needed. So, it was carried out using PFM. The value of the piezoelectric constant is $44.33 \mathrm{pm} / \mathrm{V}$. This is a significant increase in piezoelectric constant for $\mathrm{ZnO}$ nanostructure. As far our notion goes, this is the best piezoelectric constant for any kind of $\mathrm{ZnO}$ nanostructures. In our future research, we aim to enhance this coefficient further by suitable doping, applying thermal effect or interface engineering.

\section{REFERENCES}

1. D. Vanmaekelbergh and L.K.V. Vugt; ZnO nanowire lasers; Nanoscale 3, 2783 (2011)

2. M.A.M. Versteegh, D. Vanmaekelbergh and J. Dijkhuis; Room-Temperature Laser Emission of ZnO Nanowires Explained by Many-Body Theory; Phys. Rev. Lett 108, 157402 (2012)

3. I.C. Yao, P. Lin and T.Y. Seng; Nanotip fabrication of zinc oxide nanorods and their enhanced field emission properties; Nanotechnology 20, 125202 (2009)

4. G. Turner Chandler; Zinc Oxide MESFET Transistors; ME Thesis, Christchurch, University of Canterbury (2009).

5. G.Adamopoulos, S. Thomas, P.H. Wobkenberg, D.D.C. ,Bradley, M.A. McLachlan, and T.D. Anthopoulos; High-Mobility Low-Voltage $\mathrm{ZnO}$ and Li-Doped ZnO Transistors Based on ZrO2 High-k Dielectric Grown by Spray Pyrolysis in Ambient Air; Adv. Mater 23, 1894 (2011) 
6. J. Goldberger, D.J. Sirbuly, M. Law and P. Yang; ZnO Nanowire Transistors; J.Phys. Chem. B 109, 9 (2005)

7. G. Chen, C. Song, C. Chen, S. Gao, F. Zeng and F. Pan; Resistive Switching and Magnetic Modulation in Cobalt-Doped ZnO; Adv. Mater 24, 3515 (2012)

8. Y.H. Lin, M. Ying, M. Li, X. Wang and C.W. Nan; Room-temperature ferromagnetic and ferroelectric behavior in polycrystalline ZnO-based thin films; Appl. Phys. Lett 90, 222110 (2007)

9. Y.C. Yang, C. Song, X.H. Wang, F. Zeng and F. Pan; Giant piezoelectric d33 coefficient in ferroelectric vanadium doped ZnO films; Appl. Phys. Lett 92, 012907 (2008)

10. M.K. Gupta and B.J. Kumar; High Tc ferroelectricity in V-doped ZnO nanorods; Mater. Chem 21, 14559 (2011)

11. R. Yu, C. Pan and Z.L. Wang; High performance of $\mathrm{ZnO}$ nanowire protein sensors enhanced by the piezotronic effect; Energy Environ. Sci 6, 494 (2013)

12. M. Yano, K. Koike, K.I. Ogata, T. Nogami, S. Tanabe and S. Sasa; Zinc oxide-based biosensors; Phys. Status Solidi C 9, 1570 (2012)

13. L. Wang, Y. Kang, X. Liu, S. Zhang, W. Huang and S. Wang; ZnO nanorod gas sensor for ethanol detection; Sens. Actuators, B 162, 237 (2012)

14. M. Sucheaa, S. Christoulakis, K. Moschovis, N. Katsarakis and G.,Kiriakidis; ZnO transparent thin films for gas sensor applications; Thin Solid Films 515, 551 (2006)

15. Q. Zhang, C.S. Dandeneau, X. Zhou and G. Cao; ZnO Nanostructures for Dye-Sensitized Solar Cells; Adv. Mater 21, 4087 (2009) 
16. J. Seongho, S.H. Sang, N. Kushagra, S.A. Campbell and E.S. Aydil; An analysis of temperature dependent current-voltage characteristics of $\mathrm{Cu}_{2} \mathrm{O}-\mathrm{ZnO}$ heterojunction solar cells; Thin Solid Films 519, 6613 (2011)

17. M.Y. Choi, D. Choi, M.J. Jin, I. Kim, S.Y. Kim, J.Y. Choi, S.Y. Lee, J.M. Kim and S.W. Kim; Mechanically Powered Transparent Flexible Charge-Generating Nanodevices with Piezoelectric ZnO Nanorods; Adv. Mater 21, 2185 (2009)

18. Z.L. Wang; Towards Self-Powered Nanosystems: From Nanogenerators to Nanopiezotronics; Adv. Funct. Mater 18, 3553 (2008)

19. D. Pradhan, D.K. Mohapatra, S. Tymen, M. Misra and K.T. Leung; MorphologyControlled ZnO Nanomaterials for Enhanced Photoelectrochemical Performance; Mater. Express 1, 59 (2011)

20. D. Bekermann, A. Gasparotto, D. Barreca, L. Bovo, A. Devi, R.A. Fischer, O.I. Lebedev, C. Maccato, E. Tondello and G. Van Tendeloo; Highly oriented ZnO Nanorod Arrays by a Novel Plasma Chemical Vapor Deposition Process; Cryst. Growth Des; 10, 2011 (2010)

21. P.C. Chang, Z. Fan, D. Wang, W.Y. Tseng, W.A. Chiou, J. Hong and G.Luj; ZnO Nanowires Synthesized by Vapor Trapping CVD Method; Chem. Mater 16, 5133 (2004)

22. J. Conradt, J. Sartor, C. Thiele, F.M. Flaig, J. Fallert, H. Kalt, R. Schneider, M. Fotouhi, P. Pfundstein, V. Zibat and D. Gerthsen; Catalyst-Free Growth of Zinc Oxide Nanorod Arrays on Sputtered Aluminum-Doped Zinc Oxide for Photovoltaic Applications; J. Phys. Chem. C 115, 3539 (2011) 
23. P. Yang, H. Yan, S. Mao, R. Russo, J. Johnson, R. Saykally, N. Morris, J. Pham, R. He, and H.J. Choi; Controlled Growth of ZnO Nanowires and Their Optical Properties; Adv. Funct. Mater 12, 323 (2002)

24. W.I. Park, D.H. Kim, S.W. Jung and G.C. Yi; Metalorganic vapor-phase epitaxial growth of vertically well-aligned ZnO nanorods; Appl. Phys. Lett 80, 4232 (2002)

25. D. Chakraborti. Novel diluted magnetic semiconductor materials based on zinc oxide, UMI Dissertation Publishing (2011).

26. D. Pradhan and K.T. Leung; Controlled Growth of Two-Dimensional and OneDimensional ZnO Nanostructures on Indium Tin Oxide Coated Glass by Direct Electrodeposition; Langmuir 2008, 24, 9707 (2008)

27. D. Pradhan and K.T. Leung; Vertical Growth of Two-Dimensional Zinc Oxide Nanostructures on ITO-Coated Glass: Effects of Deposition Temperature and Deposition Time; J. Phys. Chem. C 112, 1357 (2008)

28. M.R. Laskar, T. Ganguli, A.A. Rahman, A. Mukherjee, N. Hatui, M.R Gokhale and A. Bhattacharya; Distorted wurtzite unit cells: Determination of lattice parameters of nonpolar a-plane AlGaN and estimation of solid phase Al content; J. Appl. Phys 109, 013107 (2011)

29. A.L. Patterson; The Diffraction of X-Rays by Small Crystalline Particles; Phys. Rev 56, 978 (1939) 
30. Y.I. Kim, K. Page and R. Seshadri; Synchrotron x-ray study of polycrystalline wurtzite Zn1-xMgxO (0 > x < 0.15): Evolution of crystal structure and polarization; Appl. Phys.

Lett 90, 101904 (2007)

31. L.Z. Xu, Y.L. Liu, H.B. Zhou, L.H. Liu, Y. Zhang and G.H. Lu; Ideal strengths, structure transitions, and bonding properties of a $\mathrm{ZnO}$ single crystal under tension; J. Phys.: Condens. Matter 21, 495402 (2009)

32. M. Stössel, J. Staudigel, F. Steuber, J. Simmerer and A. Winnacker; Impact of the cathode metal work function on the performance of vacuum-deposited organic light emitting-devices; Appl. Phys. A 68, 387 (1999)

33. S. Ju, S. Kim, S. Mohammadi and D.B. Janes; Interface studies of ZnO nanowire transistors using low-frequency noise and temperature-dependent I-V measurements; Appl. Phys. Lett 92, 022104 (2008)

34. Schroder DK; Semiconductor Material and Device Characterization; John Wiley \& Sons, New York (1998).

35. D. A. Scrymgeour and J. W. P. Hsu; Correlated Piezoelectric and Electrical Properties in Individual ZnO Nanorods; Nano Lett 8, 2204 (2008)

36. M.H. Zhao, Z.L. Wang and S. X. Mao; Piezoelectric Characterization of Individual Zinc Oxide Nanobelt Probed by Piezoresponse Force Microscope; Nano Lett 4, 587 (2004) 
37. M. Ghosh and G.M. Rao; Interstitial Defects Induced Ferroelectricity in Undoped ZnO Nanorods at Room Temperature; Sci. Adv. Mater. 5, 733 (2013)

38. R. Agrawal and H.D. Espinosa; Giant Piezoelectric Size Effects in Zinc Oxide and Gallium Nitride Nanowires. A First Principles Investigation; Nano Lett 11, 786 (2011) 